\title{
Chrominance Compensation for Multi-View Video Coding
}

\author{
Min-Woo PARK ${ }^{\dagger}$, Jong-Tae PARK ${ }^{\dagger}$, Gwang-Hoon PARK ${ }^{\dagger a)}$, and Doug-Young SUH ${ }^{\dagger}$, Members
}

\begin{abstract}
SUMMARY This letter introduces a cost-effective chrominance compensation scheme. The proposed method is applied to both 'INTER $16 \times 16$ ' and 'SKIP' modes in only anchor P-pictures. By testing using JVT common test condition, simulation results show that proposed method can obtain average BD-PSNR gains for $\mathrm{U}$ and $\mathrm{V}$ as $0.14 \mathrm{~dB}$ and $0.13 \mathrm{~dB}$, respectively while maintaining almost the same BD-PSNR's for Y. For the range of low bit-rate, it is observed that average BD-PSNR gains for $\mathrm{Y}, \mathrm{U}$ and $\mathrm{V}$ are $0.14 \mathrm{~dB}, 0.49 \mathrm{~dB}$ and $0.53 \mathrm{~dB}$, respectively. Necessary computational complexity is very marginal because the number of anchor P-pictures is very small in comparison with whole coded video sequences. However it can be found that the proposed method can significantly improve the coding efficiencies of color components.
\end{abstract}

key words: multimedia system, MPEG, multi-view video coding, $3 D$ video

\section{Introduction}

The MVC (H.264 Amd. 4 Multi-view Video Coding) coding scheme is currently under standardization on JVT (ISO/IEC JTC1/SC29/WG11 and ITU-T SG16 Q.6). And the MVC coding scheme can exploit spatial (inter-view)-temporal correlations among multi-view video by using temporal prediction with hierarchical B-picture structure and inter-view prediction [1]-[4].

Figure 1 illustrates a MVC prediction structure with 8 views and GOP size as 8 . S0, S1, ., S7 denote views, and $\mathrm{T} 0, \mathrm{~T} 1, \ldots, \mathrm{T} 16$ indicate the frames in temporal direction. At T0, T8, and T16, inter-view prediction is performed between anchor pictures such that view S2 is predicted from view S0, view $\mathrm{S} 1$ is predicted from view $\mathrm{S} 0$ and also view $\mathrm{S} 2$, view $\mathrm{S} 4$ is predicted from view $\mathrm{S} 2$, view $\mathrm{S} 3$ is predicted from view $\mathrm{S} 2$ and also view $\mathrm{S} 4$, and so on.

When performing inter-view prediction, current JMVM (Joint Multi-view Video Model) only performs the illumination compensation for the luminance information (Y component) [1], thus it may not obtain optimal performance for chrominance information ( $\mathrm{U}$ and $\mathrm{V}$ components). Due to lack of process for chrominance, subjective quality degradation may frequently be observed. Subjective color degradation and its propagation can be clearly visible in the multiview video sequences that have flat or less complicated backgrounds.

Figure 2 shows an example of color degradations in

Manuscript received June 6, 2008.

Manuscript revised September 2, 2008.

${ }^{\dagger}$ The authors are with the Media Lab. College of Electronics and Information, Kyung Hee University, 1, Seocheon, Kiheung, Yongin, Kyunggi, 449-701, Korea.

a)E-mail: ghpark@ @hu.ac.kr

DOI: 10.1587/transinf.E92.D.353
Race1 QVGA video sequence. Such subjective degradations are clearly visible at region 'A' of the left image of Fig. 2. Subjective degradation of region 'A' mainly occurs due to the lack of color information (especially in V component in the figure), which is illustrated in the right image of Fig. 2. One may easily find that there are many blocking artifacts in region 'A' of the $\mathrm{V}$ component image.

Figure 3 shows an example of propagation of color degradations from view to view. When the anchor picture of view 4 (S4) is coded as the current picture (right image of Fig. 3) by referring to the anchor picture of view 2

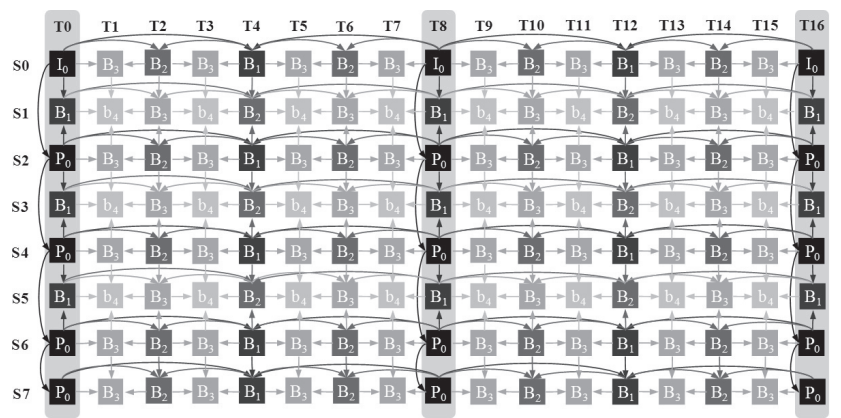

Fig. 1 Example of the prediction structure of Multi-view Video Coding $($ GOP size $=8)$.

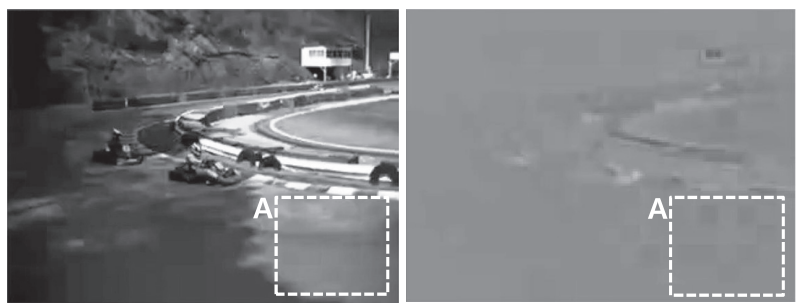

Fig. 2 Decoded anchor P-picture of Race1 QVGA sequence (right: color image, left: $\mathrm{V}$ component image).
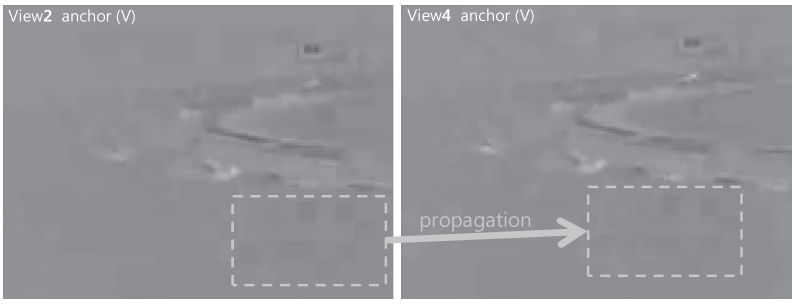

Fig. 3 Propagation of color degradation (from view 2 to view 4). 
(S2), color degradations of $\mathrm{V}$ component is propagated from view 2 picture to current view 4 picture in same pattern.

Color degradation and its propagation can also be widely visible when video plays back at low bit-rate. So, chrominance compensation is clearly needed to improve subjective quality of color information.

A cost-effective chrominance compensation scheme is introduced in this letter to improve coding efficiency for chrominance and also to minimize subjective quality degradations.

\section{Chrominance Compensation for MVC}

Visible color degradations are mainly occurred in anchor P-pictures (S2, S4, S6 and S7, at T0, T8 and T16 in Fig. 1). Anchor P-pictures are positioned in the highest level of hierarchical B-picture structure. Thus the degradations in anchor P-pictures would be strongly propagated into another anchor P-pictures, and also into anchor B-pictures and nonanchor pictures.

Proposed chrominance compensation scheme only carried out in anchor P-pictures and also it is applied in 'INTER $16 \times 16$ ' and 'SKIP' modes as same as the illumination compensation in JMVM.

In a view of process, the differences between proposed chrominance compensation scheme and the illumination compensation scheme of the JMVM are as follows.

1. Proposed chrominance compensation scheme does not encode any flag for chrominance offset.

2. Chrominance offset values are not derived in 'SKIP' modes. All offset values are differentially encoded based on the prediction of chrominance offsets from neighboring blocks.

Proposed encoding algorithm is as follows.

Step 1) If the current picture is anchor picture and also P-picture, then go to Step 2. Otherwise quit algorithm.

Step 2) If the current block is 'SKIP' mode or 'INTER $16 \times 16^{\prime}$ mode, then go to Step 3. Otherwise quit algorithm.

Step 3) Actual chrominance offset is calculated, and then goes to Step 4.

Step 4) Motion and chrominance compensation are carried out, and then go to Step 5.

Step 5) Prediction of chrominance offset is carried out and then finally its differences would be encoded.

And proposed decoding algorithm is as follows.

Step 1) If the current picture is anchor picture and also P-picture, then go to Step 2. Otherwise quit algorithm.

Step 2) If the current block is 'SKIP' mode or 'INTER $16 \times 16$ ' mode, then go to Step 3. Otherwise quit algorithm.

Step 3) Chrominance offset difference is decoded and actual offset is obtained by reverse prediction process,

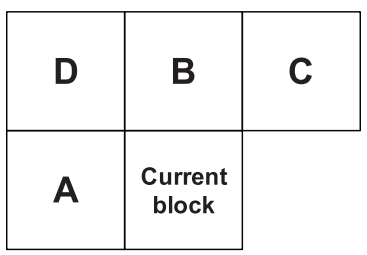

Fig. 4 Neighboring blocks for deriving the chrominance offset prediction of current block.

and then go to Step 4.

Step 4) Motion and chrominance compensation are carried out.

The calculation of the chrominance offsets for both $\mathrm{U}$ and $\mathrm{V}$ components are shown as following Eq. (1) and Eq. (2), respectively.

$$
\begin{aligned}
& \text { offset }_{c b}=\frac{1}{M \times N} \sum_{i=0}^{M} \sum_{j=0}^{N}\left\{f_{c b}(i, j)-r_{c b}(i, j)\right\} \\
& \text { offset }_{c r}=\frac{1}{M \times N} \sum_{i=0}^{M} \sum_{j=0}^{N}\left\{f_{c r}(i, j)-r_{c r}(i, j)\right\},
\end{aligned}
$$

where $M \times N$ represents the size of block, $(i, j)$ represents a position of the block, offset ${ }_{c b}$ and offset ${ }_{c r}$ represent the chrominance offsets for $\mathrm{U}$ and $\mathrm{V}$ component, $f_{c b}(i, j)$ and $f_{c r}(i, j)$ represent the pixel values of $\mathrm{U}$ and $\mathrm{V}$ of the current block, and $r_{c b}(i . j)$ and $r_{c r}(i, j)$ represent the pixel values of $\mathrm{U}$ and $\mathrm{V}$ of the reference block.

In order to compensate chrominance blocks of each $\mathrm{U}$ and V, following Eq. (3) and Eq. (4) are applied, respectively.

$$
\begin{aligned}
& r_{c b}^{\prime}=r_{c b}(i, j)+o f f s e t_{c b} \\
& r_{c r}^{\prime}=r_{c r}(i, j)+\text { offset }_{c r},
\end{aligned}
$$

where $(i, j)$ represents the position of the block, $r_{c b}^{\prime}(i, j)$ and $r_{c r}^{\prime}(i, j)$ represent the pixel values of $\mathrm{U}$ and $\mathrm{V}$ of the chrominance compensated block, and $r_{c b}(i, j)$ and $r_{c r}(i, j)$ represent the pixel values of $\mathrm{U}$ and $\mathrm{V}$ of the reference block.

Derivation process, based on neighboring blocks as shown in Fig. 4, for the chrominance offset prediction of current block is as follows.

Step 1) If all A, B and D are available and their reference picture indexes are the same as the current block's and their chrominance offsets are not equal to 0 , go to Step 2. Otherwise, go to Step 3. (If the neighboring block is neither 'INTER $16 \times 16$ ' mode nor 'P-SKIP' mode, the chrominance offset is regarded as 0. .)

Step 2) If the absolute difference of chrominance offsets between $\mathrm{A}$ and $\mathrm{D}$ is smaller than the absolute difference of chrominance offsets between A and B $(|\mathrm{D}-\mathrm{A}|<|\mathrm{D}-\mathrm{B}|)$, then chrominance offset prediction of current block is set to the chrominance offset of B block. Otherwise ( $|\mathrm{D}-\mathrm{A}| \geq|\mathrm{D}-\mathrm{B}|)$, chrominance offset prediction of current block is set to the chrominance offset of A block. 
Step 3) If all A, B and C are available and their reference picture indexes are the same as the current block's and their chrominance offsets are not equal to 0 , chrominance offset prediction of current block is set to median of the chrominance offset of A, B and C. Otherwise, go to Step 4.

Step 4) If $A$ is available and its reference picture index is the same as the current block's and its chrominance offset is not equal to 0 , then chrominance offset prediction of current block is set to the chrominance offset of A block. Otherwise, go to Step 5.

Step 5) If B is available and its reference picture index is the same as the current block's and its chrominance offset is not equal to 0 , then chrominance offset prediction of current block is set to the chrominance offset of B block. Otherwise, go to Step 6.

Step 6) If $C$ is available and its reference picture index is the same as the current block's and its chrominance offset is not equal to 0 , then chrominance offset prediction of current block is set to the chrominance offset of C block. Otherwise, go to Step 7.

Step 7) If $D$ is available and its reference picture index is the same as the current block's and its chrominance offset is not equal to 0 , then chrominance offset prediction of current block is set to the chrominance offset of D block. Otherwise, go to Step 8.

Step 8) If all A, B and C are available and their chrominance offset are not equal to 0 , then chrominance offset prediction of current block is set to median of the chrominance offset of A, B and C. Otherwise, go to Step 9.

Step 9) The chrominance offset prediction of current block is set to 0 .

\section{Simulation Results}

Proposed method is compared with JMVM 7.0.2 [5] as an anchor and also the method that the illumination compensation scheme of JMVM is simply applied on the chrominance components as it is (illustrated as CC in the table and figures), in a view of coding efficiency. Simulation is carried out based on JVT common test conditions for multi-view video coding with quantization parameter set of $\{37,32,27,22\}[6]$ and also the quantization parameter set of $\{47,42,37,32\}$ to analyze the coding performance of low bit-rate test range. Additionally, the method to encode the chrominance offset is the same method to encode the luminance offset in JMVM.

Table 1 shows the average BD-PSNR's and average BD-rates [7] of the proposed method (Prop.) and the method-CC in comparison with JMVM7.0.2 for whole multi-view video test sequences.

For the low bit-rate test range, total average PSNR gains (BD-PSNR) and bit-savings (BD-rates) of Y of the proposed method for all test sequences are $0.14 \mathrm{~dB}(1.80 \%)$, and the range of average PSNR gains and bit-savings are $-0.02 \sim 0.50 \mathrm{~dB}(-0.51 \sim 5.05 \%)$. Total average PSNR gains
Table 1 Comparisons of average BD-PSNR's and average BD-rates at both low bit-rate and JVT common test condition.

\begin{tabular}{|c|c|c|c|c|c|c|c|c|c|c|c|c|}
\hline \multicolumn{13}{|c|}{ Low bit-rate test condition: $\mathrm{QP}=\{47,42,37,32\}$} \\
\hline & & $\mathbf{Y}$ & $I$ & & & $\mathbf{U}$ & & & & $\mathbf{v}$ & & \\
\hline \multirow{2}{*}{$\begin{array}{c}\text { Test } \\
\text { sequence }\end{array}$} & \multicolumn{2}{|c|}{$\begin{array}{c}\text { BD- } \\
\text { PSNR(dB) }\end{array}$} & \multicolumn{2}{|c|}{$\begin{array}{c}\text { BD- } \\
\text { rate(\%) }\end{array}$} & \multicolumn{2}{|c|}{\begin{tabular}{c|} 
BD- \\
PSNR(dB)
\end{tabular}} & \multicolumn{2}{|c|}{$\begin{array}{c}\text { BD- } \\
\text { rate(\%) }\end{array}$} & \multicolumn{2}{|c|}{$\begin{array}{c}\text { BD- } \\
\text { PSNR(dB) }\end{array}$} & \multicolumn{2}{|c|}{$\begin{array}{c}\text { BD- } \\
\text { rate(\%) }\end{array}$} \\
\hline & Prop. & $\mathrm{CC}$ & Prop. & $\mathrm{CC}$ & Prop. & $\mathrm{CC}$ & Prop. & $\mathrm{CC}$ & Prop. & $\mathrm{CC}$ & Prop. & $\mathrm{CC}$ \\
\hline Flamenco 2 & 0.21 & -0.33 & -4.05 & 5.47 & 0.56 & 0.11 & -19.13 & -3.97 & 0.52 & 0.02 & -17.04 & -0.64 \\
\hline Breal & 2 & -0.95 & 0.51 & 19.58 & 76 & -0.19 & -24.14 & 6.34 & .08 & -0.10 & -32.72 & 3.00 \\
\hline & & -0.57 & -5.00 & 7.81 & 48 & .02 & & 0.32 & 61 & 0.07 & -18.27 & -2.29 \\
\hline Akkc & 10 & -1.09 & -0.93 & 6.54 & 8 & -0.11 & -17.55 & 5.70 & 29 & .26 & -9.37 & 9.49 \\
\hline $\mathrm{Ba}$ & 01 & -0.60 & 0.14 & 11.79 & 38 & 0.03 & -16.27 & -1.03 & .33 & .05 & -14.17 & 2.36 \\
\hline & 01 & -0.66 & -0.08 & 12.14 & 59 & 13 & -18.70 & 4.45 & .59 & 0.01 & \begin{tabular}{|l|l|}
-19.42 \\
\end{tabular} & 0.29 \\
\hline & & -1.83 & -5.05 & 21.96 & 3 & .48 & -17.87 & 14.95 & .49 & -0.48 & -16.17 & 13.49 \\
\hline $\mathrm{Ul}$ & 00 & -0.31 & 0.09 & 6.80 & & 0.02 & -10.19 & -0.69 & 0.30 & 0.12 & -13.46 & -5.6 \\
\hline Tot & 0.14 & -0.79 & -1.80 & 12.76 & 0.49 & -0 & -17.26 & 3.2 & 0.53 & -0.0 & -17.58 & 2.5 \\
\hline \multicolumn{13}{|c|}{ JVT common test condition: $\mathrm{QP}=\{37,32,27,22\}$} \\
\hline & \multicolumn{4}{|c|}{$Y$} & \multicolumn{4}{|c|}{ T } & \multicolumn{4}{|c|}{$\mathbf{V}$} \\
\hline \multirow{2}{*}{$\begin{array}{c}\text { Test } \\
\text { sequence }\end{array}$} & \multicolumn{2}{|c|}{$\begin{array}{c}\text { BD- } \\
\text { PSNR(dB) }\end{array}$} & \multicolumn{2}{|c|}{$\begin{array}{c}\text { BD- } \\
\operatorname{rate}(\%)\end{array}$} & \multicolumn{2}{|c|}{$\begin{array}{c}\text { BD- } \\
\text { PSNR(dB) }\end{array}$} & \multicolumn{2}{|c|}{$\begin{array}{c}\text { BD- } \\
\text { rate(\%) }\end{array}$} & \multicolumn{2}{|c|}{$\begin{array}{c}\text { BD- } \\
\text { PSNR(dB) }\end{array}$} & \multicolumn{2}{|c|}{$\begin{array}{c}\text { BD- } \\
\text { rate(\%) }\end{array}$} \\
\hline & Prop. & $\mathrm{CC}$ & Prop. & CC & Prop. & $\mathrm{CC}$ & Prop. & $\mathrm{CC}$ & Prop. & $\mathrm{CC}$ & Prop. & $\mathrm{CC}$ \\
\hline Flamenco2 & 0.00 & -0.12 & 0.07 & 2.64 & 0.17 & 0.09 & -5.55 & -3.09 & 0.12 & 0.00 & -3.98 & -0.12 \\
\hline Breakdan & -0.01 & -0.21 & 0.60 & 10.88 & 13 & 0.04 & -8.10 & -2.06 & 0.26 & 0.12 & \begin{tabular}{|l|}
-14.44 \\
\end{tabular} & \begin{tabular}{|l|}
-7.29 \\
\end{tabular} \\
\hline & 0.00 & \begin{tabular}{|c|}
-0.19 \\
\end{tabular} & \begin{tabular}{l|l|}
0.06 \\
\end{tabular} & 4.83 & 7 & 0.06 & \begin{tabular}{|l|l|}
-5.35 \\
\end{tabular} & \begin{tabular}{|c|}
-1.87 \\
\end{tabular} & \begin{tabular}{l|l}
0.16 \\
\end{tabular} & 0.04 & \begin{tabular}{|c|}
-5.09 \\
\end{tabular} & -1.52 \\
\hline Akk & 01 & -0. & 0.25 & 7.37 & 3 & 0. & -6.15 & \begin{tabular}{|l|}
-0.72 \\
\end{tabular} & 0.06 & $\begin{array}{l}-0.09 \\
\end{array}$ & -2.22 & 3.40 \\
\hline Ballroon & \begin{tabular}{|c|}
-0.01 \\
\end{tabular} & \begin{tabular}{|c|}
-0.17 \\
\end{tabular} & \begin{tabular}{l|l}
0.23 \\
\end{tabular} & 4.61 & \begin{tabular}{ll|}
0.13 & \\
\end{tabular} & \begin{tabular}{l|l|}
0.10 \\
\end{tabular} & \begin{tabular}{|l|}
-5.03 \\
\end{tabular} & $\begin{array}{l}-4.02 \\
\end{array}$ & \begin{tabular}{l|l|}
0.09 \\
\end{tabular} & 0.02 & \begin{tabular}{|l|}
-3.43 \\
\end{tabular} & -0.90 \\
\hline Exit & -0.02 & -0.16 & 0.60 & 6.37 & 0.15 & 0.05 & $\begin{array}{l}-7.33 \\
\end{array}$ & -2.56 & 0.15 & 0.05 & \begin{tabular}{|c|}
-7.45 \\
\end{tabular} & -2.80 \\
\hline $\mathrm{Re}$ & 0.00 & \begin{tabular}{|c|}
-0.45 \\
\end{tabular} & 0.10 & 10.32 & 0.11 & \begin{tabular}{|c|}
-0.14 \\
\end{tabular} & -4.02 & 5.75 & \begin{tabular}{l|l|}
0.09 \\
\end{tabular} & $\begin{array}{l}-0.16 \\
\end{array}$ & -3.25 & 6.41 \\
\hline & 0.00 & -0.11 & 0.07 & 2.93 & 0.06 & 0.03 & -2.41 & -1.00 & \begin{tabular}{l|l}
0.09 \\
\end{tabular} & 0.06 & \begin{tabular}{|c|}
-2.97 \\
\end{tabular} & \begin{tabular}{|l|}
-2.19 \\
\end{tabular} \\
\hline $\begin{array}{c}\text { Total } \\
\text { average }\end{array}$ & 07 & -0.22 & 0.25 & 6.24 & 0.14 & 0.03 & -5.49 & -1.20 & 0.13 & 0.01 & -5.35 & -0.63 \\
\hline
\end{tabular}

and bit-savings of chrominance ( $\mathrm{U}$ and $\mathrm{V}$ ) of the proposed method for all test sequences are $0.49 \mathrm{~dB}(17.26 \%)$ and $0.53 \mathrm{~dB}(17.58 \%)$, respectively. The range of average PSNR gains and bit-savings of the proposed method for $\mathrm{U}$ are $0.18 \sim 0.76 \mathrm{~dB}(10.19 \sim 24.14 \%)$ and the range of average PSNR gains and bit-savings of the proposed method for $\mathrm{V}$ are $0.29 \sim 1.08 \mathrm{~dB}(9.37 \sim 32.72 \%)$. At the JVT common test condition, total average PSNR of the proposed method for Y is almost same with JMVM 7.0.2. Total average PSNR gains and bit-savings of the proposed method for $\mathrm{U}$ and $\mathrm{V}$ are $0.14 \mathrm{~dB}(5.49 \%)$ and $0.13 \mathrm{~dB}(5.35 \%)$, respectively. The range of average PSNR gains and bit-savings of $U$ of the proposed method are $0.06 \sim 0.17 \mathrm{~dB}(2.41 \sim 8.10 \%)$ and the range of average PSNR gains and bit-savings of $\mathrm{V}$ of the proposed method are $0.06 \sim 0.26 \mathrm{~dB}(2.22 \sim 14.44 \%)$.

The coding performance of the method-CC is far lower than the proposed scheme in a view of average BD-PSNR gains and bit-savings, because the method-CC may need so many bits to encode the flags and offsets to compensate the color components.

The RD-curves for all Y, U and $\mathrm{V}$ components in "Breakdancers" of JMVM 7.0.2, the proposed scheme and the conventional chrominance compensation method are shown in Fig. 5. In case of Y (luminance), the proposed scheme has the almost same coding performances as the JMVM 7.0.2 at the JVT common test condition, and even improves the coding performance at the low bit-rate test range. Moreover, the proposed scheme obtains definitely notable improvement of coding efficiencies in comparison with the JMVM 7.0.2, especially for the color components $(\mathrm{U}$ and $\mathrm{V})$. The coding performances of $\mathrm{U}$ and $\mathrm{V}$ of the method-CC at the high bit-rate range are slightly better than the proposed method. However in the rest parts of bit-rate range, the coding performances of the method-CC for all components (Y, U, and $\mathrm{V}$ ) are far lower than the proposed 

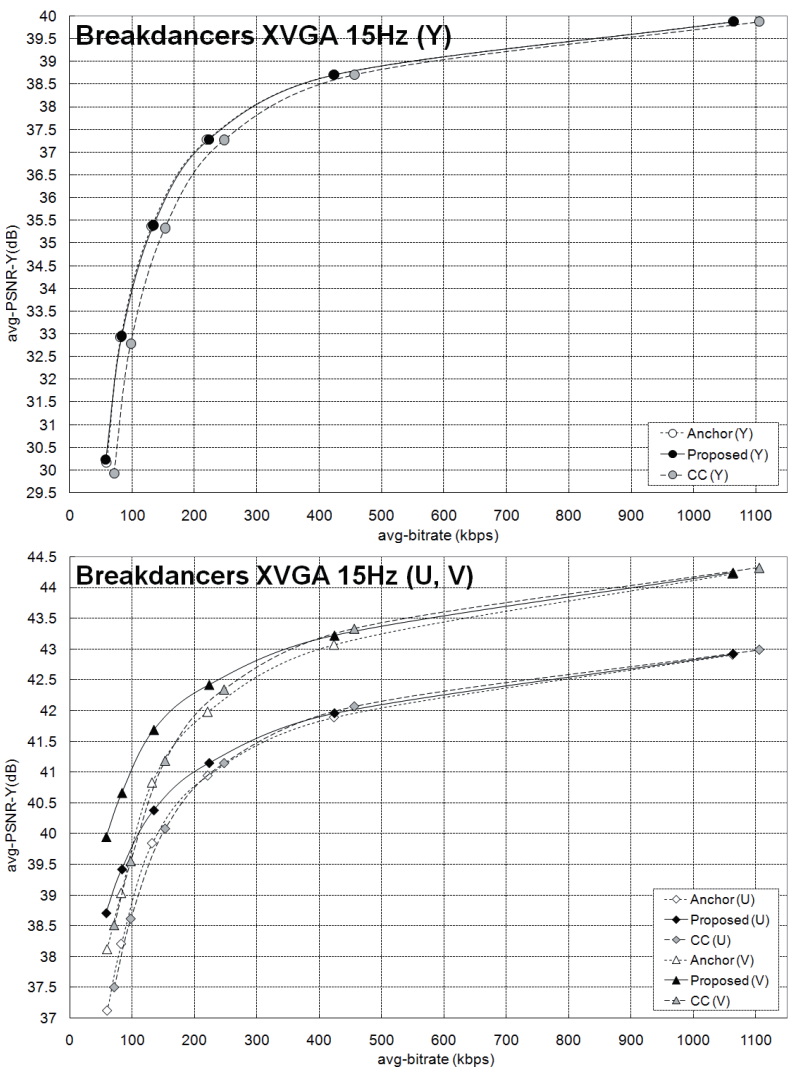

Fig. 5 Comparisons of RD-curves in "Breakdancers."

scheme.

In terms of computational complexity, since the number of anchor P-pictures is very small in comparison with whole coded multi-view video sequences, necessary computational complexity is very marginal.

\section{Conclusions}

In this letter, MVC chrominance compensation is introduced to improve coding efficiencies of the chrominance and thus to reduce the subjective quality degradations. Proposed scheme is applied to both 'INTER $16 \times 16$ ' and 'SKIP' modes in ONLY anchor P-pictures. Necessary computational complexity is very marginal because the number of anchor P-pictures is a very small portion in comparison with the whole video sequences. However simulation results show that the proposed scheme can significantly improve the coding efficiencies of color components.

\section{Acknowledgements}

This work was supported by the Korea Science and Engineering Foundation (KOSEF) grant funded by the Korea government (MEST) (No. R0A-2005-000-10061-0).

\section{References}

[1] A. Vetro, P. Pandit, H. Kimata, and A. Smolic, "Joint Multiview Video Model (JMVM) 7.0," Joint Videa Team (JVT) of ISO/IEC JTC1/SC29/WG11 and ITU-T SG16 Q.6, JVT-Z207, Turkey, Jan. 2008.

[2] A. Vetro, P. Pandit, H. Kimata, and A. Smolic, "Joint Draft 6.0 on Multiview Video Coding," Joint Video Team (JVT) of ISO/IEC JTC1/SC29/WG11 and ITU-T SG16 Q.6, JVT-Z209, Turkey, Jan. 2008.

[3] ITU-T and ISO/IEC JTC1, "Advanced Video Coding for Generic Audiovisual Services," ITU-T Recommendation H.264-ISO/IEC 14496-10 AVC, 2003.

[4] H. Schwarz, D. Marpe, and T. Wiegand, "Hierarchical B pictures," Joint Video Team (JVT) of ISO/IEC JTC1/SC29/WG11 and ITU-T SG16 Q.6, JVT-P014, Poland, July 2005.

[5] P. Pandit, A. Vetro, and Y. Chen, "JMVM 7 software," Joint Video Team (JVT) of ISO/IEC JTC1/SC29/WG11 and ITU-T SG16 Q.6, JVT-Z208, Turkey, Jan. 2008.

[6] A. Su, A. Vetro, and A. Smolic, "Common Test Conditions for Multiview Video Coding," Joint Video Team (JVT) of ISO/IEC JTC1/SC29/WG11 and ITU-T SG16 Q.6, JVT-U211, China, Oct. 2006.

[7] G. Bjontegaard, "Calculation of average PSNR differences between RD-curves," ITU-T SG16 Q.6, VCEG-M033, Texas, USA, April 2001. 